\title{
Qualidade de folhas e rendimento de óleo essencial em hortelã pimenta (Mentha $x$ Piperita L.) submetida ao processo de secagem em secador de leito fixo
}

\author{
GASPARIN, P.P1*; ALVES, N.C.C².; CHRIST,D³.; COELHO, S.R.M4. \\ 1,2,3,4 Universidade Estadual do Oeste do Paraná - UNIOESTE, Rua Universitária, 2069, CEP: 85819-110, Cascavel \\ - Brasil *matematicapri@gmail.com
}

\begin{abstract}
RESUMO: O uso de plantas medicinais e seus derivados para o tratamento de doenças é uma prática antiga e se percebe, atualmente, uma crescente procura por produtos naturais, incluindo medicamentos, produtos alimentícios, e cosméticos. Hortelã pimenta (Mentha x Piperita L), além de ser uma planta medicinal, pode ser utilizada para obtenção de aromatizantes, infusões, e temperos. O processo de secagem se faz necessário para aumentar o tempo de conservação e a vida útil do produto facilitando seu transporte, manuseio, e armazenamento. Para que os produtos derivados da hortelã pimenta tenham qualidade é necessário estudos sobre o processo de pré e de pós-colheita. Objetivou-se neste estudo identificar a temperatura de secagem, em duas velocidades de ar circulante, que minimize a degradação da cor das folhas e permita obter maior rendimento de óleo essencial. Folhas da hortelã foram colhidas manualmente no horário entre 7:15 e 8:00, e submetidas a secagem em duas velocidades do ar $\left(0,3\right.$ e 0,5 m.s. $\left.{ }^{-1}\right)$ e em cinco temperaturas $\left(30,40,50,60\right.$ e $\left.70^{\circ} \mathrm{C}\right)$. O material seco foi analisado quanto à cor utilizando-se colorímetro com escala do sistema CIELab baseado em coloração dentro dos padrões da Norma DIN 6174 (1979). A quantificação do óleo essencial foi realizada por hidrodestilação em aparelho Clevenger. Observou-se que temperaturas superiores a 50 ${ }^{\circ} \mathrm{C}$ reduzem o rendimento. $\mathrm{A}$ cor das folhas submetidas à secagem diferencia-se da cor das folhas frescas. De acordo com a Norma DIN 6174, a cor das folhas submetidas a secagem com temperatura até $40^{\circ} \mathrm{C}$ são "Facilmente distinguíveis" e para as folhas submetidas à secagem a temperaturas superior a $50^{\circ} \mathrm{C}$, a diferenciação é "Muito grande". Conclui-se que para obter o máximo de rendimento do óleo essencial e o mínimo de degradação da cor, a secagem deve ser realizada a temperatura de até $50^{\circ} \mathrm{C}$. A velocidade do ar de secagem, na faixa de $0,3 \mathrm{a}$ 0,5 m.s.1, não afetou os parâmetros avaliados.
\end{abstract}

Palavras-chave: cor, óleo essencial, planta medicinal.

ABSTRACT: Quality of leaves and essential oil yield of peppermint (Mentha x piperita L.) submitted to the drying process in fixed-bed dryer. The use of medicinal plants and their derivatives for the treatment of diseases is an ancient practice. Currently, there is a growing demand for natural products, not only medicines, but also food and cosmetics. The peppermint (Mentha $x$ piperita L.), in addition to being a medicinal plant, can be used for obtaining flavorings, spices and tea infusions. The drying process is necessary to increase the shelf life of the product and to facilitatg itr transport, handling and storage. For peppermint derivatives of sufficient quality, it is necessary studies on the pre- and post-harvest. The objective of this study is to identify the temperature and air velocity that minimizs the degradation of the color of the leaves with higher oil yield. PepperMint leaves were harvested manually from 7:15 to 8:00 a.m. and dried in trays in a completely randomized design in a $2 \times 5$ factorial design, being two air velocities $(0.3$ and

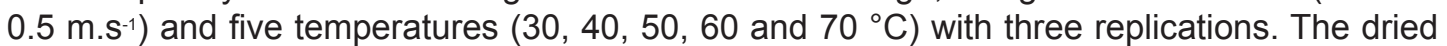
material was analyzed for color using a colorimeter with the CIELab scale system based on a color within the DIN 6174 standards (1979). The quantification of the essential oil was performed by hydrodistillation in a Clevenger-type apparatus. The analyzes were performed in triplicate. Ws observed that the yield of essential oil of the dry leaves was highertwhan compared to the yield presented by the fresh leaves. This effect was attributed to the fact that the lower water content in the leaves enables the vapor stream generated in the extractor to promots a more effective drag of the volatile oilcompounds stored in the cells; however, a temperature exceeding $50{ }^{\circ} \mathrm{C}$ 
promotes a reduction in yield. The color of the leaves submitted to drying is different from the color of fresh leaves. According to the DIN 6174 standard, the color of the leaves subjected to drying at the temperature up to $40^{\circ} \mathrm{C}$ is "easily distinguishable" and, for the leaves submitted to drying at temperatures higher than $50^{\circ} \mathrm{C}$, the differentiation is "too large". We conclude that to get the maximum yield of essential oil and minimal color degradation, drying should be carried out at a temperature of up to $50^{\circ} \mathrm{C}$. The rate of the drying processes in the rangeofrom 0.3 to $0.5 \mathrm{~m}^{-\mathrm{s}^{-1}}$ did not affect the parameters evaluated.

Keywords: color, essential oil, medicinal plant

\section{INTRODUÇÃO}

O hortelã pimenta, pertencente ao gênero Mentha à família Lamiaceae, apresenta-se amplamente plantas distribuíds em todo o mund, tendo, como centro de origem, a Europa meridional e a região do Mediterrâneo, suporto temperaturas baixas, mas é melhor adaptads ao clima tropical. Possum folhas, pecioladas e pubescentes, com flores de coloração lilás ou branca, reunidas em espigas nas axilas das folhas (Mattos, 2000).

A Mentha é usada para fins medicinais tais como, analgésico estomacal e intestinal, estimulante das funções cardíacas, controle da azia, gastrite, cólicas e gases (Grisi et al., 2006).

O principal produto das plantas do gênero Mentha é o óleo essencial, normalmente rico em mentoo. Porém, Outras substâncias podem alterar as qualidades sensoriais do óleo essencial ou ainda terem valor comercial por serem utilizadas nas indústrias alimentícias e farmacêuticas (Paulus et al., 2007). As aplicações industriais do mentol podem ser encontradas em produtos de higiene bucal, flavorizantes, aromatizantes de alimentos e bebidas, em perfumarias, confeitarias, e produtos farmacêuticos (Domijan et al., 2005).

Estes óleos são produzidos e armazenados em estruturas chamadas tricomas glandulares que estão distribuídos preferencialmente na face abaxial das folhas, podendo ocorrer também na face adaxial, em menor densidade (Deschampset al., 2006). As técnicas mais empregadas para a extração de óleos essenciais são: extração por arraste a vapor, hidrodestilação, extração com solventes orgânicos, prensagem a frio e extração com fluído super crítico (Bruneton, 2001, Simões \& Spitzer, 2003).

Lorenzi \& Matos (2002) relataram que nas espécies medicinais a secagem é uma operação unitária de preparação para o armazenamento a fim de atender às necessidades da indústria farmacêutica de fitoterápicos, que não tem estrutura para usar as plantas frescas nas quantidades exigidas para a produção industrial.

Como parâmetros de qualidade para plantas medicinais e aromáticas pode-se citar a quantidade e qualidade dos princípios ativos, as características sensoriais (sabor e odor), as características visuais (aparência e coloração), e a contaminação microbiana (Allong et al., 2000).

Um dos parâmetros da qualidade é $s$ característics visuas, que podm ser avaliads utilizando instrumentos, tais como: colorímetros e espectrofotômetros. A vantagem de se utilizar esses instrumentos é a eliminação do aspecto subjetivo da avaliação visual (Martinazzo et al., 2008).

Outro método, é através da análise da cor pel sistema CIELAB 1976, que atualmente apresenta maior aceitação, send o mais indicado para a percepção sensorial (Alonso-Salces et al., 2005)eO sistema CIELAB mede o grau de luminosidade $\left(L^{*}\right)$, que é a relação entre a luz refletida absorvida, a cromaticidade vermelha/verde $\left( \pm a^{*}\right)$, e cromaticidade amarela/azul $\left( \pm b^{*}\right)$. A cor representada no sistema de coordenadas retangulares $\left(L^{*}, a^{*}, b^{*}\right)$, mostra variáveis dependentes que seriam de difícil interpretaçã, s avaliadas separadamente, A transformação em coordenadas polares, ângulo de coloração ou tom $\left(\mathrm{H}^{*}\right)$ e índice de croma ou cromaticidade $\left(C^{*}\right)$ é, portanto, a maneira mais apropriada para a interpretação dos resultados).

O objetivo do presente estudo foi identificar a temperatura e a velocidade do ar de secagem para se obter o mínimo de degradação da cor e o máximo de rendimento do óleo essencial das folhas de M. x piperita.

\section{MATERIAL E MÉTODOS}

O experimento foi realizado no campus da Universidade Estadual do Oeste do Paraná (UNIOESTE), em Cascavel, PR. As plantas utilizadas foram M. x piperita. as quais foram cultivadas em canteiros e conduzidas em sistema orgânico, com adubação orgânica. $O$ transplante das mudas de $M$. $x$ piperita foi realizado em junho de 2011.

A colheita das folhas de M. x piperita, foi realizada em duas etapas, sendo a primeira realizada aos oitenta e cinco dias após o transplante (14/09/11), e a segunda, dois dias depois (16/09/11) seguindo as especificações da Embrapa (2007).

As folhas foram colhidas manualmente com auxílio de tesoura de poda no horário das 7:15 às

Rev. Bras. PI. Med., Campinas, v.16, n.2, supl. I, p.337-344, 2014. 
8:00 horas, devido à maior concentração do óleo essencial nestes horários.

Antes de iniciar s processs de secagem, o material coletado foi selecionado para obtenção das folhas integras e sadias e acondicionado em sacos plásticos e armazenados em B.O.D. a $5^{\circ}$, por duas horas, no máximo. $O$ teor de água da $M$. x piperita foi determinado imediatamente após a coleta e ao final do processo de secagem, empregando-se o método gravimétrico.

Para a realização da secagem das folha, utilizou-se secador de laboratórioo de camada fixa com bandejao. Na parte superior do secador foram colocadas três bandejas quadradas removíveis de $361 \mathrm{~cm}^{2}$ pe perfuradas com malha de $2,75 \mathrm{~mm}$. Para a insuflação do ar utilizou-se ventilador centrifugo com motor de $1 \mathrm{cV}$.

Foi utilizado o delineamento estatístico inteiramente casualizado, em esquema fatorial $2 \times 5$, sendo duas velocidades do ar $\left(0,3\right.$ e $\left.0,5 \mathrm{~m} \mathrm{~s}^{-1}\right)$ e cinco temperaturas $\left(30,40,50,60\right.$ e $\left.70^{\circ} \mathrm{C}\right)$, com três repetições. Durante a secagem foram realizadas pesagens periódicas, até se atingir o teor de água final de 0,11 b.s. Imediatamente após a secagem as folhas, estas foram armazenadas em recipiente de vidrs vedads com parafilme e papel alumínio, e armazenadas em B.O.D a $5{ }^{\circ} \mathrm{C}$, conforme descrito por Venskutonis (1997).

O aquecimento do ar foi obtido por meio de três resistências elétricas, sendo duas ligadas diretamente na rede de energia e a terceira ligada a um controlador responsável pelo seu acionamento quando a temperatura de secagem estivesse abaixo ou acima do valor especificado. A umidade relativa do ar foi monitorad, por meio de um psicrômetro de bulbo molhado.

A qualidade do produto seco foi analisada quadto a cor e quantificação do teor de óleo essencial e comparada com a planta fresca antes da secagem.

A cor foi determinada por leitura direta de reflectância das coordenadas $L^{*}, a^{*}$ e $b^{*}$ empregando a escala CIELAB em colorímetro tristímulo para o iluminante $10 \% D_{65}$, o colorímetro utilizado foi o CR-410 da marca Konica Minolta, e as medições foram realizadas em triplicatas.

O ângulo de coloração ou tom $\left(\mathrm{H}^{*}\right)$ é o aspecto da cor mais familiar que pode ser descrito, pois identifica cores como vermelho, verde, azul ou amarelo. Inicia no eixo $+a^{*}$ e é expresso em graus: $0^{\circ}$ para vermelho $\left(+a^{*}\right), 90^{\circ}$ para amarelo $\left(+b^{*}\right), 180^{\circ}$ para verde $\left(-a^{*}\right)$ e $270^{\circ}$ para azul $\left(-b^{*}\right)$. O índice de croma $\left(C^{*}\right)$ indica a intensidade ou pureza do tom, independente de quão clara ou escura é a cor. Quanto maior é o seu valor, a cor é mais intensa ou altamente cromática parecendo luminosa ou concentrada, enquanto que valores baixos (acromático) indicam cor acinzentada, fraca ou diluída (Hill et al., 1997, Gonnet, 1998). Para o cálculo do ângulo de coloração e do índice de croma, foram utilizadas as equações 1 e 2 respectivamente.

$$
\begin{gathered}
H^{*}=\tan ^{-1}\left(\frac{b^{*}}{a^{*}}\right) \\
\left.C^{*}=\sqrt{\left[\left(a^{*}\right)^{2}+\left(b^{*}\right)^{2}\right.}\right]
\end{gathered}
$$

A localização das cores das amostras no espaço colorimétrico e até mesmo a estatística não são suficientes para expressar se as diferenças de cor são possíveis de serem distinguidas visualmente. Estas diferenças de cor podem ser calculadas pelas distancias entre dois pontos no espaço tridimensional $(\Delta E)$ definido pelos parâmetros $a^{*}, b^{*}$ e $L^{*}$. Matematicamente, o parâmetro colorimétrico $\Delta \mathrm{E}$ é descrito pela equação 3 .

$$
\Delta E_{d b}=\sqrt{\left(\Delta L^{*}\right)^{2}+\left(\Delta a^{*}\right)^{2}+\left(\Delta b^{*}\right)^{2}}
$$

A extração do óleo essencial foi realizada por meio de hidrodestilação, utilizando-se o aparelho de Clevenger, adaptado a um balão de fundo redondo com capacidade de $500 \mathrm{~mL}$ (Ming et al.,1996) com aquecimento mantido na temperatura mínima necessária para ebulição. Para a hidrodestilação foram utilizadas aproximadamente $40 \mathrm{~g}$ de amostra de folhas secas (resultado da secagem) sendo o tempo de cada destilação de 5 horas, contados do momento da condensação da primeira gota, segundo recomendações de Zenebon et al. (2008).

O cálculo do rendimento em porcentagem foi realizado segundo Zenebon et al. (2008), como a razão entre o número de $\mathrm{mL}$ de óleo essencial destilado, pela massa em gramas da amostra, multiplicada por cem. Os dados foram submetidos à análise de variância (ANOVA) e suas médias comparadas através do teste Tukey a $5 \%$ de probabilidade utilizando o software Sisvar (Ferreira, 2010).

\section{RESULTADO E DISCUSSÃO}

\section{Rendimento do óleo essencial}

A análise de variância dos dados de rendimento do óleo essencial de folhas de Mentha $x$ Piperita L. em relação à matéria seca quando 
submetidas à secagem em diferentes temperaturas e velocidade do ar é apresentada na Tabela 1.

Na Figura 1 é apresentado o rendimento de óleo essencial em função da temperatura do ar de secagem para as duas velocidades do ar.

De acordo com a Figura 1, para todas as temperaturas avaliadas, o rendimento do óleo essencial foi menor quando utilizado a velocidade do ar de $0,3 \mathrm{~m} \cdot \mathrm{s}^{-1}$, contudo pela análise de variância (Tabela 1), este tratamento não foi suficiente para apresentar diferença estatisticamente significativa a $5 \%$ de probabilidade.

O rendimento de óleo essencial das folhas frescas $(0,26 \%)$ foi inferior quando comparado ao rendimento apresentado pelas folhas secas, diferindo estatisticamente a $5 \%$ de probabilidade pelo teste de Tukey. Para Martins (2000) o menor conteúdo de água nas folhas, após a secagem, permite que a corrente de vapor gerada no extrator possa arrastar mais eficientemente as substâncias voláteis armazenadas nas células, quando comparado com o material verde. Segundo Guenther (1972), devido ao alto teor de umidade nas plantas frescas, há forte tendência à aglutinação do óleo, impedindo que o vapor penetre de forma mais uniforme nos tecidos vegetais.

De acordo com a Figura 1, a secagem a temperatura de $50{ }^{\circ} \mathrm{C}$ foi que apresentou maior

TABELA 1. Análise de variância dos dados obtidos de rendimento do óleo essencial de folhas de Mentha $x$ Piperita L. secas sob diferentes temperaturas e velocidades do ar

\begin{tabular}{llllll}
\hline FV & GL & SQ & QM & Fc & Pr>Fc \\
\hline Temperatura & 4 & 0,183748 & 0,045937 & 27,010 & $0,0037^{*}$ \\
Velocidade do ar & 1 & 0,007840 & 0,007840 & 4,610 & $0,0983^{\text {ns }}$ \\
Erro & 4 & 0,006802 & 0,001701 & & \\
Total & 9 & 0,1983990 & & &
\end{tabular}

$\mathrm{CV}(\%)=3,48$

* significativa a $5 \%$ de probabilidade.

ns - não significativa a $5 \%$ de probabilidade.

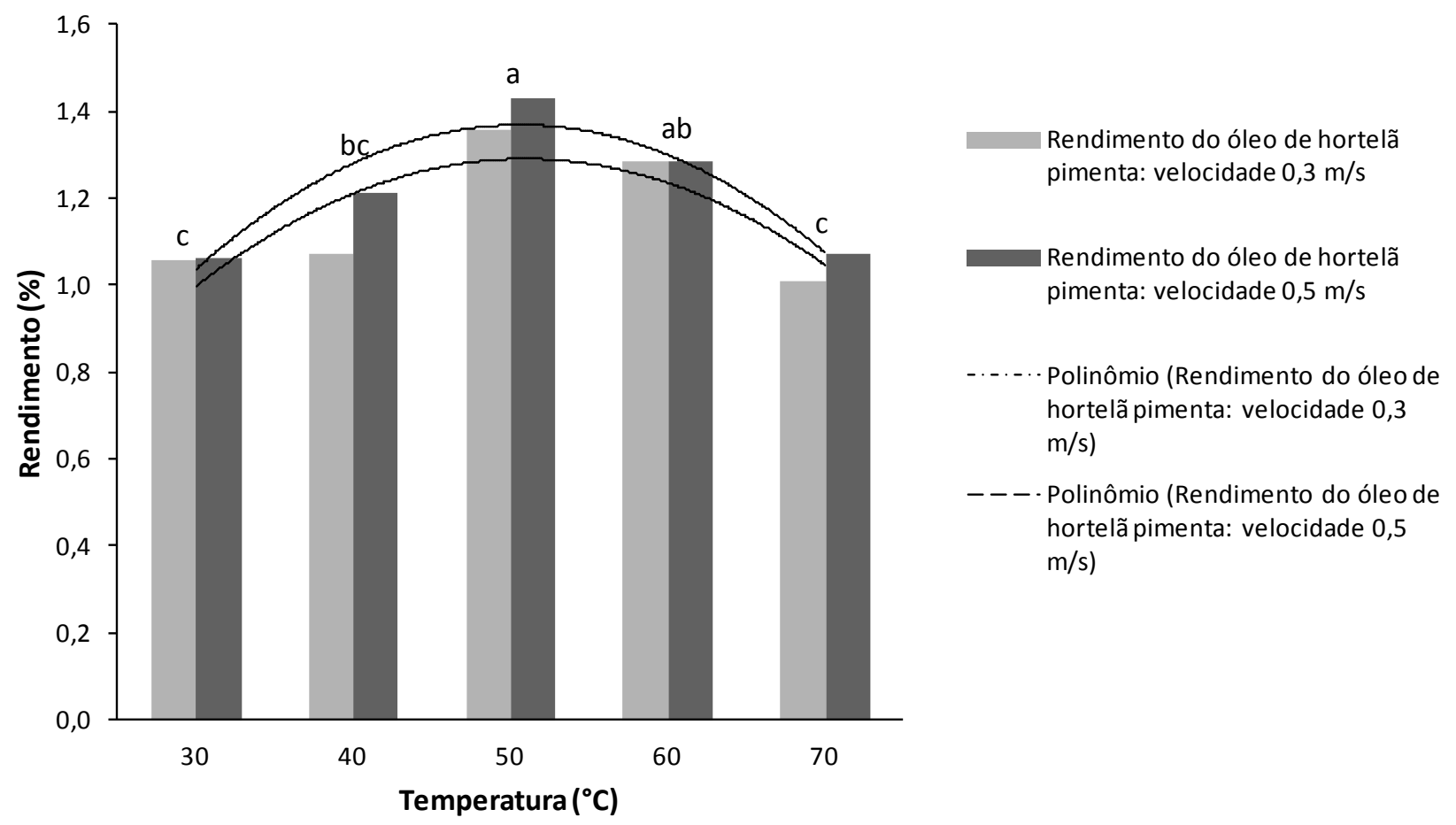

FIGURA 1. Rendimento do óleo essencial, em \% relativa à matéria seca, de lâminas foliares de Mentha $x$ Piperita L. em diferentes temperaturas $\left(T_{1}=30 ; T_{2}=40 ; T_{1}=50 ; T_{1}=60 ; T_{1}=70\right)$ e diferentes velocidades de $\operatorname{ar}\left(0,3 \mathrm{~m} \mathrm{~s}^{-1}\right.$ e 0,5 $\left.\mathrm{m} \mathrm{s}^{-1}\right)$. Letras iguais não diferem estatisticamente entre si pelo teste de Tukey $(P<0,05)$ a $5 \%$ de probabilidade. 
rendimento de óleo essencial, $1,35 \%\left(0,3 \mathrm{~m} . \mathrm{s}^{-1}\right) \mathrm{e}$ $1,42 \%\left(0,5 \mathrm{~m}^{\mathrm{s}} \mathrm{s}^{-1}\right)$, porém, a média foi considerada estatisticamente igual ao rendimento obtido quando as folhas foram secas a temperatura de $60^{\circ} \mathrm{C}$, com rendimento de $1,28 \%$ para as duas velocidades do ar utilizadas na secagem. $O$ rendimento de óleo essencial do material seco a temperatura de $40{ }^{\circ} \mathrm{C}$ foi de $1,07 \%\left(0,3{\mathrm{~m} . \mathrm{s}^{-1}}^{-1}\right.$ e $1,21 \%\left(0,5 \mathrm{~m} . \mathrm{s}^{-1}\right)$, não diferindo estatisticamente do rendimento apresentado pelas folhas secas a $60^{\circ} \mathrm{C}$.

Os resultados encontrados estão de acordo com Radunz et al. (2006), os quais verificaram que até ser atingida a temperatura de $50{ }^{\circ} \mathrm{C}$, ocorreaumento gradual no rendimento do óleo essencial, e que após, com temperaturas superiores, o rendimento decresce na mesma proporção. Os autores ainda argumentam que este comportamento possa estar relacionado com a temperatura de volatilização dos componentes do óleo essencial, pois em Mentha, o óleo essencial se localiza em estruturas mais sensíveis, tais como pêlos glandulares e glândulas epidérmicas. Além disso, valores de temperatura do ar de secagem superiores a $50{ }^{\circ} \mathrm{C}$ podem ter causado danos às estruturas secretoras e armazenadoras de óleo essencial do produto seco.

Blanco et al. (2002) concluíram que o teor de óleo essencial foi significativamente influenciado pela temperatura do ar de secagem, quando avaliaram o efeito de três temperaturas, 40,60 e 80 ${ }^{\circ} \mathrm{C}$, no processo de secagem da da Mentha x Piperita L., que foi acondicionada em sacos de papel kraft, em estufa com circulação forçada de ar.

Buggle et al. (1999) obteve resultados semelhantes quando realizaram a secagem do capim limão (Cymbopogon citratus) em forno aquecido a $30,50,70$ e $90{ }^{\circ} \mathrm{C}$. Com relação ao óleo essencial, obtiveram como melhor rendimento $1,43 \%$ à temperatura de secagem de $50^{\circ} \mathrm{C}$.

Radünz et al. (2010) avaliaram a influência da temperatura do ar de secagem sobre o teor e composição do óleo essencial extraído de guaco
(Mikania glomerata Spreng). Seis tratamentos de secagem foram usados: ar ambiente e ar aquecido a $40,50,60,70$ e $80^{\circ} \mathrm{C}$. Os maiores teores de óleo essencial de guaco foram obtidos com o ar de secagem a $50^{\circ} \mathrm{C}$.

Pode-se observar que a maioria dos trabalhos realizados com relação à secagem de plantas medicinais os autores concluíram que a temperatura de secagem influencia o rendimento do óleo essencial, ou seja, se faz necessário saber qual a melhor temperatura de secagem para obter-se o maior rendimento de óleo essencial preservando seus componentes químicos. De forma prática, essas informações são importantes principalmente para as coorperativas e empresas que realizam este tipo de trabalho.

\section{Influência do processo de secagem na coloração}

A avaliação de cada atributo do sistema CIELAB em função do tratamento de secagem (diferentes temperaturas e velocidades de ar0,30 $\mathrm{m} \cdot \mathrm{s}^{-1}$ e $0,50 \mathrm{~m} \cdot \mathrm{s}^{-1}$ ) em comparação com a planta fresca é apresentada na Tabela 2.

Os parâmetros de avaliação da cor das folhas da Mentha $x$ Piperita L. (Tabela 2) quando submetida à secagem utilizando as velocidades do ar de 0,3 e 0,5 m.s-1, não apresentaram diferença estatisticamente significativa a $5 \%$ de probabilidade pela ANOVA (não apresentada), indicando que estas velocidades não foram suficientes para apresentar alterações neste parâmetro.

Com relação à planta fresca é possível observar na Tabela 2 que a coordenada $a^{*}$ apresenta coloração esverdeada com luminosidade $\left(L^{*}\right)$ um pouco escura e com índice de croma $\left(\mathrm{C}^{*}\right)$, com coloração opaca.

Os valores das coordenadas com relação à Luminosidade $\left(L^{*}\right)$ varia de 0 a 100 , sendo escura apróxima de 0 e branca a a próxima de 100. Não houve diferença estatística neste parâmetro entre as folhas frescas e para o

TABELA 2. Médias dos parâmetros utilizando a escala CIELab, para análise da cor da Mentha x Piperita

\begin{tabular}{lllllllllll}
\hline & \multicolumn{1}{l}{ Coordenadas } \\
\cline { 2 - 11 } Tratamentos & $\mathrm{a}^{*}$ & \multicolumn{1}{l}{$\mathrm{b}^{*}$} & \multicolumn{1}{l}{$\mathrm{L}^{*}$} & \multicolumn{3}{l}{$\mathrm{H}^{*}$} & \multicolumn{3}{l}{$\mathrm{C}^{*}$} \\
\hline Folhas frescas & $-5,54 \mathrm{~d}$ & & $11,32 \mathrm{~d}$ & & $38,84 \mathrm{~b}$ & & $116,06 \mathrm{c}$ & $12,61 \mathrm{~d}$ \\
\hline Velocidadens & $0,3 \mathrm{~m} . \mathrm{s}$ & $0,5 \mathrm{~m} . \mathrm{s}$ & $0,3 \mathrm{~m} . \mathrm{s}$ & $0,5 \mathrm{~m} . \mathrm{s}$ & $0,3 \mathrm{~m} . \mathrm{s}$ & $0,5 \mathrm{~m} . \mathrm{s}$ & $0,3 \mathrm{~m} . \mathrm{s}$ & $0,5 \mathrm{~m} . \mathrm{s}$ & $0,3 \mathrm{~m} . \mathrm{s}$ & $0,5 \mathrm{~m} . \mathrm{s}$ \\
\hline $30^{\circ} \mathrm{C}^{* *}$ & $-1,50 \mathrm{a}$ & $-2,08 \mathrm{a}$ & $9,39 \mathrm{c}$ & $9,86 \mathrm{c}$ & $38,56 \mathrm{~b}$ & $38,47 \mathrm{~b}$ & $99,10 \mathrm{a}$ & $101,95 \mathrm{a}$ & $9,50 \mathrm{c}$ & $10,08 \mathrm{c}$ \\
$40^{\circ} \mathrm{C}^{* *}$ & $-1,63 \mathrm{ab}$ & $1,55 \mathrm{ab}$ & $9,29 \mathrm{c}$ & $9,56 \mathrm{c}$ & $37,71 \mathrm{~b}$ & $38,50 \mathrm{~b}$ & $99,92 \mathrm{a}$ & $99,23 \mathrm{a}$ & $9,43 \mathrm{bc}$ & $9,68 \mathrm{bc}$ \\
$50^{\circ} \mathrm{C}^{* *}$ & $-0,37 \mathrm{bc}$ & $0,66 \mathrm{bc}$ & $8,51 \mathrm{bc}$ & $7,96 \mathrm{bc}$ & $37,35 \mathrm{~b}$ & $36,59 \mathrm{~b}$ & $92,47 \mathrm{a}$ & $85,26 \mathrm{a}$ & $8,52 \mathrm{ab}$ & $7,99 \mathrm{ab}$ \\
$60^{\circ} \mathrm{C}^{* *}$ & $1,58 \mathrm{c}$ & $1,81 \mathrm{c}$ & $7,16 \mathrm{ab}$ & $6,78 \mathrm{ab}$ & $35,34 \mathrm{a}$ & $34,74 \mathrm{a}$ & $77,59 \mathrm{~b}$ & $75,02 \mathrm{~b}$ & $7,33 \mathrm{a}$ & $7,02 \mathrm{a}$ \\
$70^{\circ} \mathrm{C}^{* *}$ & $1,86 \mathrm{c}$ & $1,73 \mathrm{c}$ & $6,95 \mathrm{a}$ & $6,99 \mathrm{a}$ & $34,94 \mathrm{a}$ & $34,58 \mathrm{a}$ & $75,02 \mathrm{~b}$ & $75,25 \mathrm{~b}$ & $7,20 \mathrm{a}$ & $6,81 \mathrm{a}$ \\
\hline
\end{tabular}

**Letras iguais nas colunas não diferem estatisticamente entre si pelo teste de Tukey $(P<0,05)$ a $5 \%$ de probabilidade.

ns $O$ fator velocidade do ar de secagem foi não significativo a $5 \%$ de probabilidade pela ANOVA (não apresentada).

Rev. Bras. PI. Med., Campinas, v.16, n.2, supl. I, p.337-344, 2014. 
material seco nas temperaturas de 30,40 e $50^{\circ} \mathrm{C}$ , apresentando luminosidade escura. Quando a secagem foi efetuada nas temperaturas de 60 e $70{ }^{\circ} \mathrm{C}$, o parâmetro $L^{*}$ continuou não apresentando diferença estatística entre estas temperaturas, mas diferiu das folhas frescas e das folhas secas até a temperatura de $50{ }^{\circ} \mathrm{C}$, indicando que a secagem realizada a temperaturas superior a $50{ }^{\circ} \mathrm{C}$ causam escurecimento nas folhas.

Para a coordenada $a^{*}$, valores negativos refletem a cor verde da folha, passando para o vermelho quando se tornam positivos. Nos dados apresentados na Tabela 2 é possível observar menores valores para as folhas frescas, diferindo estatisticamente das folhas secas. Para as folhas secas, quanto maior a temperatura do ar de secagem utilizada, maior o valor apresentado, ou seja, as cores próximas do verde das folhas frescas se tornam avermelhadas quanto maior a temperatura do ar de secagem.

Para a coordenada $b^{*}$ os valores encontrados foram todos positivos, o que significa cor amarela (clara), sendo o maior valor observado para as folhas frescas e que se diferenciaram estatisticamente das folhas secas. Quando as folhas são submetidas a secagem, os valores da coordenada $b^{*}$ decrescem em função do aumento da temperatura. Valores menores indicam tendência ao azul (escuro), ou seja, ao aumentar a temperatura do ar de secagem, a coloração das folhas fica mais escura.

O ângulo de matiz $\left(\mathrm{H}^{*}\right)$ se refere ao quadrante do ciclo colorimétrico onde se encontrará a união das cores, para, então, definir qual foi a coloração das folhas antes e após a realização da secagem.

De acordo com o apresentado na Tabela 2, o maior valor apresentado foi para as folhas frescas (verde), diferenciando-se estatisticamente das folhas submetidas a secagem. Quando submetido à secagem as temperaturas 30,40 e $50^{\circ} \mathrm{C}$, não apresentaram diferença estatística, da mesma forma para as temperaturas 60 e $70^{\circ} \mathrm{C}$. Observa-se que as temperaturas 50,60 e $70^{\circ} \mathrm{C}$ encontram-se no 1. quadrante do sistema CIELAB 1976. As temperaturas 60 e $70^{\circ} \mathrm{C}$ apresentam coloração mais avermelhada, isto significa que, sob temperaturas elevadas, a secagem danifica mais intensamente a cor da folha. As temperaturas 30 e $40^{\circ} \mathrm{C}$, que se encontram no $2^{\circ}$ quadrante, apresentam coloração mais próxima da cor natural das folhas de hortelãpimenta, ou seja, mais esverdeada.

O índice de croma $\left(\mathrm{C}^{*}\right)$ indica intensidade ou pureza do tom. Maiores valores, a cor é mais cromática e possui brilho e valores menores a cor é acromática e opaca. O valor apresentado pelas folhas frescas (Tabela 2) foi maior, diferenciandose estatisticamente das folhas frescas quando submetidas à secagem. Para as folhas submetidas à secagem, decréscimos nos valores foram observados com o aumento da temperatura do ar de secagem, sendo considerado como índice acromático (cor opaca). Pelas médias apresentadas na Tabela 2 é possível observar que para as temperaturas 30 e 40 ${ }^{\circ} \mathrm{C}$ não houve diferença estatística, assim como para as temperaturas 40 e $50^{\circ} \mathrm{C}$, e para as temperaturas 50,60 e $70^{\circ} \mathrm{C}$.

\section{Diferença de cor}

A análise de variância para a Diferença de Cor $\left(\Delta \mathrm{E}_{\mathrm{ab}}\right)$ das folhas frescas em relação às folhas secas em função da temperatura e da velocidade do ar de secagem demonstrou que a velocidade do ar de secagem não afetou este parâmetro, desta forma, avaliou-se separadamente a variável temperatura por meio do teste de médias, apresentada na Tabela 3.

De acordo com o apresentado na Tabela 3 , nota-se que a diferença de cor das folhas submetidas à secagem em relação às folhas frescas, calculado pela equação (3), cresce em função do aumento da temperatura do ar de secagem. Folhas submetidas à secagem nas temperaturas 30 e 40 -C não apresentaram diferença estatística, assim como para as temperaturas 40 e $50{ }^{\circ} \mathrm{C}$ e para as temperaturas 60 e $70{ }^{\circ} \mathrm{C}$.

Para avaliar a diferença de cor utilizou-se a

TABELA 3. Teste de média para a diferença de cor $\left(\Delta \mathrm{E}_{\mathrm{ab}}\right)$, das folhas secas em diferentes temperaturas e velocidades do ar de secagem em relação às folhas frescas

\begin{tabular}{ccc}
\hline \multirow{2}{*}{ Temperatura $\left({ }^{\circ} \mathrm{C}\right)$} & \multicolumn{2}{c}{ Velocidade do ar de secagem $\left(\mathrm{m} \cdot \mathrm{s}^{-1}\right)^{\mathrm{ns}}$} \\
\cline { 2 - 3 } & $0,3^{*}$ & $0,5^{*}$ \\
\hline 30 & $4,486 \mathrm{a}$ & $3,974 \mathrm{a}$ \\
40 & $4,548 \mathrm{ab}$ & $4,374 \mathrm{ab}$ \\
60 & $6,07 \mathrm{~b}$ & $7,402 \mathrm{~b}$ \\
70 & $8,958 \mathrm{c}$ & $9,562 \mathrm{c}$ \\
\hline
\end{tabular}

* Médias seguidas de mesma letra na coluna não diferem entre si pelo teste de Tukey $(P<0,05)$.

ns $O$ fator velocidade do ar de secagem foi não significativo a $5 \%$ de probabilidade pela ANOVA (não apresentada).

Rev. Bras. PI. Med., Campinas, v.16, n.2, supl. I, p.337-344, 2014. 
TABELA 4. Percepção humana para diferentes valores de $\Delta \mathrm{E}_{\mathrm{ab}}$ de acordo com a norma DIN 6174 , (1979)

\begin{tabular}{ll}
\hline Diferenças $\Delta \mathrm{E}_{\mathrm{ab}}$ & Classificação \\
\hline $0,0-0,2$ & Imperceptível \\
$0,2-0,5$ & Muito pequena \\
$0,5-1,5$ & Pequena \\
$1,5-3,0$ & Distinguível \\
$3,0-6,0$ & Facilmente Distinguível \\
Maior que 6,0 & Muito grande \\
\hline
\end{tabular}

Fonte: Norma DIN 6174 (1979)

Tabela 4 da norma DIN 6174 (1979) que estabelece relação aos valores de $\Delta \mathrm{E}$ para a percepção do olho humano. De modo geral, diferenças de cor em duas amostras justapostas, podem ser distinguidas em valores de $\Delta \mathrm{E}$ acima de 0,2-0,5 (Silva et al., 2007).

Ao aumentar a temperatura do ar de secagem, maior será o impacto sobre a coloração das folhas e maior será a distinção da cor entre as folhas da planta fresca e a cor das folhas da planta seca. Comparando os valores da diferença de cor apresentados na Tabela 3 e a escala da Tabela 4 , nota-se que as folhas submetidas à secagem com temperatura até $40{ }^{\circ} \mathrm{C}$ são "Facilmente distinguíveis" e para as folhas submetidas à secagem a temperaturas superior a $50^{\circ} \mathrm{C}$, a diferenciação da cor passa a ser "Muito grande".

Como a coloração é considerada um parâmetro de qualidade, o consumidor ao observar uma cor muito escura das folhas, poderá considerar que ao utilizar estas folhas como fonte para um tratamento, possivelmente não farão o efeito esperado, pois com altas temperaturas além de danificar a coloração também pode comprometer o princípio ativo da planta.

\section{CONCLUSÃO}

Em função dos resultados obtidos, pôde-se concluir que para obter o máximo de rendimento do óleo essencial e o mínimo de degradação da cor durante a secagem de folhas de M. x Piperita, esta deve ser realizada a temperatura de até 50 ${ }^{\circ} \mathrm{C}$, independentemente da velocidade do ar de secagem, na faixa de $0,30 \mathrm{~m} \cdot \mathrm{s}^{-1}$ a $0,50 \mathrm{~m} \cdot \mathrm{s}^{-1}$.

\section{AGRADECIMENTO} financeiro.

Os autores agradecem ao CNPq pelo apoio

AUniversidade Estadual do Oeste do Paraná (UNIOESTE) e a Coordenação de Aperfeiçoamento de Pessoal de Nível Superior (CAPES) pelo apoio financeiro indispensável na execução do presente trabalho.

\section{REFERÊNCIA}

ALLONG, R. et al. The effect of cultivar, fruit ripeness, storage temperature and duration on quality of freshcut mango. Acta Horticulturae, v. 10, n. 509, p.487 - 494, 2000.

ALONSO-SALCES, R. M. et al. Chemometric classification of Basque and French ciders based on their total polyphenol contents and CIELAB parameters. Food Chemistry, v.91, n.1, p.91-98, 2005.

BLANCO, M.C.S.G. et al. Drying temperatureeffects in peppermint essential oil content and composition. Acta Horticulturae, n.569, p.95-98, 2002.

BUGGLE, V. et al. Influence of different drying temperatures on the amount of essential oils and citral content in Cymbopogon citrates (DC) Stapf. Poaceae. Acta Horticulturae, n.500, p.71-74, 1999.

BRUNETON, J. Farmacognosia. 2 ed. Zaragoza, Espanha: Acribia S.A, 2001. 594p.

DESCHAMPS, C.; et al.Densidade de tricomas glandulares e produção de óleo essencial em Mentha arvensis L., Mentha x piperita L. e Mentha cf. aquatica L. Ciência e Natura, v. 28, n. 1, p. 23-34, 2006.

DIN - Deutsche Institut für Normung; DIN 6174. Farbmetrische Bestimmung Von Farbabständen bei Körperfarben nach der CIELAB- Formel, Beuth Verlag, Berlim und Köln, 1979.

DOMIJAN, A.M. et al. Seed borne fungi and ochratoxina: A contamination of dry beans (Phaseolus vulgaris L.). Food and Chemical Toxicology, v. 43, n.3, p. 427432, 2005.

EMPRESABRASILEIRADE PESQUISAAGROPECUÁRIA - EMBRAPA. Recomendações técnicas para o cultivo de hortaliças em agricultura familiar. Brasília, 2007. (Embrapa Hortaliças, Circular Técnica. n. 47).

FERREIRA, D. F. Sisvar: versão 5.3 (Build 73). Lavras: Departamento de Ciências Exatas, Universidade Federal de Lavras, 2010.

GONNET, J. F. Colour effects of co-pigmentation of anthocyanins revisited-1. A colorimetric definition using the CIELAB scale. Food Chemistry, v.63, n.3, p.409415, 1998.

GRISI, M. C. M. et al. Avaliação de genótipos de Menta (Mentha spp) nas condições do Distrito Federal, Brasil. Revista Brasileira de Plantas Medicinais, v. 8, n. 4, p. 33-39, 2006.

GUENTHER, E. The essential oils. 6 ed. Huntington, N.Y.: R.E. Krieger, 1972, 63 p.

HILL, B. et al. Comparative analysis of the quantization of color spaces on the basis of the CIELAB color-difference formula. ACM Transactions on Graphics, v.16, n.2, p.109-154, 1997.

LORENZI,H.; MATOS,F.J.A. Plantas medicinais no Brasil: Nativas e exóticas. Nova Odessa - SP:Instituto Plantarum, 2002. 512p.

MARTINAZZO, A.P. et al.Avaliação colorimétrica de folhas secas de Cymbopogon citratus (D.C) Stapf durante o armazenamento em diferentes embalagens. Revista Brasileira de Produtos Agroindustriais, v.10, n.2,

Rev. Bras. PI. Med., Campinas, v.16, n.2, supl. I, p.337-344, 2014. 
p.131-140, 2008.

MARTINS, P. M. Influência da temperatura e velocidade do ar de secagem no teor e na composição química do óleo essencial de capim limão (Cymbopogum citratus (D.C.) STAPF). 2000. 77 p. Dissertação (Mestrado- Área de Concentração em Plantas Medicinais) -Departamento de Engenharia Agrícola Plantas Medicinais, Universidade Federal de Viçosa, Viçosa.

MATTOS, S.H. Perspectivas do cultivo de plantas medicinais para a fitoterapia no Estado do Ceará. Horticultura Brasileira, v.18, p.45-46, 2000.

MING, L. C. et al. Yield of essential oil of and citral content in different parts of lemongrass leaves (Cymbopogon citratus D.C.) tapf.) Poaceae. Acta Horticulturae, n. 426, p. 555-559, 1996.

PAULUS, D. et al. E.Teor e qualidade do óleo essencial de menta (Mentha arvensis L.) produzida sob cultivo hidropônico e em solo.RevistaBrasileira de Plantas Medicinais, v.9, n.2, p.80-87, 2007.

RADÜNZ, L.L. et al. Influência da temperatura do ar de secagem no rendimento do óleo essencial de hortelã comum (MENTHAX VILOSA HUNDS). Engenharia na Agricultura, v.14, n.4, p.250 - 257, 2006.

RADÜNZ, L.L. et al.Study of essential oil from guaco leaves submitted to different drying air temperature. Engenharia na Agricultura, v.18, p.241-247. 2010.

SILVA, F. et al. Isotermas de dessorção de Calendula officinalis L.: determinação experimental e modelagem matemática. Revista Brasileira de Plantas Medicinais, v.9, n.1, p.21-28, 2007.

SIMÕES, C.M.; SPITZER,V.Óleos voláteis. In: Farmacognosia da planta ao medicamento. SIMÕES, C.M. (Org). 5 ed. Porto Alegre, Florianópolis: UFRGS/ UFSC, 2003, cap.18, 467 - 495p.

VENSKUTONIS, P.R. Effect of drying on the volatile constituents of thyme(Thymus vulgaris L.) and sage (Salvia officinalis L.).Food Chemistry, v. 59, n.2, p.219227, 1997.

ZENEBON, O. et al. Métodos físico-químicos para análise de alimentos. In: Condimentos e vinagres. São Paulo: Instituto Adolfo Lutz, 2008. p. 888-889. 